\title{
Seasonal Community Succession of the Phyllosphere Microbiome
}

\author{
Julia K. Copeland, ${ }^{1}$ Lijie Yuan, ${ }^{2}$ Mehdi Layeghifard, ${ }^{1}$ Pauline W. Wang, ${ }^{1,2}$ and David S. Guttman ${ }^{1,2}$ \\ ${ }^{1}$ Department of Cell and Systems Biology, and ${ }^{2}$ Centre for the Analysis of Genome Evolution and Function, University \\ of Toronto, 25 Willcocks Street, Toronto, Ontario, M5S 3B2, Canada
}

Submitted 22 October 2014. Accepted 2 December 2014.

The leaf microbiome is influenced by both biotic and abiotic factors. Currently, we know little about the relative importance of these factors in determining microbiota composition and dynamics. To explore this issue, we collected weekly leaf samples over a 98-day growing season from multiple cultivars of common bean, soybean, and canola planted at three locations in Ontario, Canada, and performed Illumina-based microbiome analysis. We find that the leaf microbiota at the beginning of the season is very strongly influenced by the soil microbiota but, as the season progresses, it differentiates, becomes significantly less diverse, and transitions to having a greater proportion of leaf-specific taxa that are shared among all samples. A phylogenetic investigation of communities by reconstruction of unobserved states imputation of microbiome function inferred from the taxonomic data found significant differences between the soil and leaf microbiome, with a significant enrichment of motility gene categories in the former and metabolic gene categories in the latter. A network co-occurrence analysis identified two highly connected clusters as well as subclusters of putative pathogens and growth-promoting bacteria. These data reveal some of the complex ecological dynamics that occur in microbial communities over the course of a growing season and highlight the importance of community succession.

A diverse and abundant community of nonpathogenic microbes naturally exists in the phyllosphere (Vorholt 2012). The entire microbial community contains viruses, bacteria, filamentous fungi, yeasts, algae, and, occasionally, protozoa and nematodes (Lindow and Brandl 2003). Of the cellular organisms, bacteria are by far the most abundant members of this community, with a typical cell density of $10^{6}$ to $10^{7}$ cells $/ \mathrm{cm}^{2}$ of leaf tissue (Lindow and Brandl 2003; Vorholt 2012). These bacteria can interact with the plant negatively by causing disease, positively by producing or modifying metabolites and hormones or interfering with pathogen growth, or have a neutral, commensal interaction (Lindow and Brandl 2003). Characterizing the composition and dynamics of the phyllosphere microbiome is a key step toward understanding how the community may influence plant health and development.

Corresponding author: D. S. Guttman; E-mail: david.guttman@utoronto.ca

*The $\boldsymbol{e}$-Xtra logo stands for "electronic extra" and indicates that six supplementary figures and five supplementary tables are published online.

(C) 2015 The American Phytopathological Society
There are many factors controlling the composition of the phyllosphere microbiota. Some factors are dynamic, such as environmental variables, including radiation, temperature, humidity, pollution, and nutrient availability, while other factors are more stable and constant, such as host genotype and geographical location (Vorholt 2012). Previous studies have identified certain factors that play a dominant role in structuring the microbiota; however, these results are often contradictory between studies. In some instances, host genotype has been shown to be the primary factor driving the composition of the phyllosphere microbiome (de Oliveira Costa et al. 2012; Kim et al. 2012) whereas, in other cases, geographic location has had the greatest influence on community composition (Finkel et al. 2011; Rastogi et al. 2012; Redford et al. 2010). Perennial plants often have season-dependent communities that are highly similar from year to year (Redford and Fierer 2009), while other perennial plant communities change dramatically from one year to another and have high season variability (Jackson and Denney 2010). Phyllosphere communities of sterile, media-grown young plants have shown similarities to airborne communities (Maignien et al. 2014), whereas soilgrown plants often have phyllosphere communities similar to soil communities (Bodenhausen et al. 2013; Hardoim et al. 2011; Perazzolli et al. 2014). Even demographic factors such as migration, birth, and death can play a significant role (Vorholt 2012). The overarching conclusion from prior studies is, not surprisingly, that the plant phyllosphere is very complex, and the relative influence of environmental and biological factors on the composition of the community remains unclear when all these factors are considered simultaneously.

The bacterial microbiome is typically characterized using the 16S ribosomal RNA (rRNA) gene (Wang and Qian 2009). Within the 16S rRNA gene there are nine hypervariable regions. The high level of sequence variation within these regions, or a combination of regions, provides taxonomic resolution that is often comparable with that achieved using whole 16S rRNA gene sequences (Chakravorty et al. 2008; Maughan et al. 2012). Unfortunately for plant microbiome research, homology between chloroplast and bacterial 16S rRNA genes made amplification and sequencing exclusively of the bacterial 16S rRNA gene difficult. Previous research characterizing the phyllophere community of the common bean and soybean required using culture-dependent techniques (de Oliveira Costa et al. 2012; Kuklinsky-Sobral et al. 2004). However, recent improvements in methodology using polymerase chain reaction (PCR) primers designed to amplify the V5, V6, and V7 hypervariable regions of the $16 \mathrm{~S}$ rRNA gene allow amplification specifically of bacterial DNA while excluding amplification of chloroplast DNA (Bulgarelli et al. 2012; Chelius and Triplett 2001). These innovations have allowed further investigation of the endophy- 
tic and epiphytic leaf-associated microbiome in a thorough, culture-independent manner.

In this study, we aim to explore how biotic and abiotic factors affect the community structure of the phyllosphere microbiome. We investigated the bacterial communities associated with three agricultural crop species commonly farmed in Ontario, Canada: Glycine max (soybean), Brassica napus (canola), and Phaseolus vulgaris (common bean). Common garden experiments using multiple cultivars of these species were grown at multiple locations and leaves were harvested throughout a 98day growing season. This allowed us to investigate colonization of the plant after development of true leaves, until leaf senescence at the end of the growing season.

\section{RESULTS}

The leaf microbiome is influenced by many environmental and biological factors, including location, weather, plant development, seasonal changes, and host genotype. The effects of these factors on the microbiome were examined simultaneously by sampling leaves from a variety of plant genotypes, grown at multiple locations, over the course of a 98-day growing season in Ontario, Canada. The V5 to V7 hypervariable regions of the 16S rRNA gene were amplified and sequenced using the Illumina MiSeq. Over nine sampling weeks, 103 leaf microbiome samples were collected, amplified, and sequenced. Four sequencing runs resulted in a total of 10,146,432 sequences retained for analysis after stringent quality filtering and contig assembly. Sequences shorter or longer than the expected amplicons size and chimeras were also removed, yielding 8,856,423 sequences kept for clustering. This produced an average of 48,930 $\pm 33,266$ high-quality sequences per sample. Sequences were clustered at $97 \%$ identity and binned into operational taxonomic units (OTU). Singletons and very low-abundance OTU $(<0.005 \%$ relative abundance [RA]) were removed from the analysis to control for sequencing errors (Bokulich et al. 2013), resulting in a total of 1,556 high-confidence OTU. Any genus with an RA less than $0.1 \%$ per sample was removed from the analysis based on our single-species control (Maughan et al. 2012). The chloroplastexcluding primers amplified primarily bacterial 16S rRNA genes straight from ground leaf tissues, with only minimal coamplification of chloroplast or mitochondria averaging 0.236 and $0.260 \%$ per sample, respectively.

We initially assessed the RA of bacteria in the community at the phylum level for associations with sampling date, plot location, stage of development, plant species, and plant cultivar (Fig. 1A). A high RA of Actinobacteria and Proteobacteria was observed in both plant and soil samples. Leaf samples also had moderately high amounts of Firmicutes, Bacteroidetes, Thermi, and Chloroflexi. WS3, TM6, and Planctomycetes were found only in soil whereas Fusobacterium was found only in leaves.

A nonparametric analysis of variance indicates that, overall, the RA of phyla were significantly different $(P<0.05)$ between soil and leaf communities and different for the leaf communities between sampling dates and developmental stages but did not differ between plot location, host species, or any of their respective cultivars (Supplementary Table S1a). The RA of soil community phyla did not differ significantly between sampling dates or plot location. All phyla except Bacteroidetes had significantly different RA between plant and soil samples. Within communities that did not experience overall change, many RA of phyla differed individually but not enough to contribute significantly to the community.

We also assessed the distribution of RA at the genus level and found that the leaf community followed the same general patterns of significant differences, with significant differences found between sampling date and developmental stage. At the genus level, host species and bean cultivars also showed significantly different RA of genera, whereas plot location and soybean cultivars did not differ significantly.

\section{Diversity decreases during the growing season.}

Principal coordinate analysis ( $\mathrm{PCoA}$ ) was performed using the weighted UniFrac $\beta$ diversity metric on the OTU data to visualize multivariate distances between samples. OTU data rarefied to the lowest 10 th percentile of sequences $(7,994$ sequences). PCoA plots show a strong separation of plant samples from soil samples on principal coordinate axis 1 (PC1), while PC2 separates by sampling date, developmental stage of the plant, and most abundant genus (Fig. 1B). Plot location, host species, and host cultivar do not have an observable effect on positioning in the PCoA plot (Supplementary Fig. S1).

Species richness and evenness (Shannon diversity index) and phylogenetic diversity (PD) index were calculated using the rarefied OTU data. Both measures of diversity reduced significantly from the beginning of the growing season to the end $(P<0.005)$. PD increased moderately during the second and third week of sampling, followed by a steady decrease for the remaining weeks (Fig. 1C). Sampling date significantly predicted Shannon diversity and PD $\left(P<0.005, R^{2}=0.492\right.$, and $P<0.005, R^{2}=0.422$, respectively), whereas location and host species did not predict either diversity measure (Supplementary Table S2). Developmental stage was omitted from the analysis because it was highly correlated with sampling date (adjusted $R^{2}=0.856, P<0.005$ ).

Position of the sample on PC1 and PC2 (Fig. 1B) can be significantly predicted by sampling date, developmental stage of the plant, Shannon diversity index, and PD index (Supplementary Table S3).

\section{Community succession over time.}

The majority of the community was composed of a small number of highly abundant genera (>1\% RA). These abundant genera were from the phyla Actinobacteria, Proteobacteria, Firmicutes, and Thermi (Fig. 2A). Total precipitation and specific rainfall events both had a strong influence on the microbiome. The greatest amount of daily rainfall for the entire sampling season occurred on 31 July 2012. A large amount of rainfall also occurred from 25 to 26 July 2012(Fig. 2B). These rainfall events coincided with a dramatic change in the microbiome between 25 July and 1 August 2012. During this time, the community shifted from being Oxalobacteraceae (Other) dominated to being Frigoribacterium, Geodermatophilus, or Kineosporiaceae (Other) dominated (Fig. 2C). Other genera from the orders Actinomycetales, Bacillales, and Gaiellales displayed significant changes in RA (Table 1). Changes in plant development also coincided with these incidents of rainfall, because the first appearance of mature, yellowing seed was noted on bean plants on 1 August 2012.

Communities were grouped by host species to determine whether changes in the microbiome were a reflection of plant maturity, precipitation, or overall seasonal changes and community succession (Fig. 2D). Bean plants developed more quickly than soybean or canola but the shift from Oxalobacteraceae (Other) dominance to a genus of Actinobacteria dominance was observed first in soybean. The initial change in community structure was observed in bean and soybean prior to these incidents of heavy rainfall; only canola showed a dramatic change in community structure following the rain (Fig. 2D). Canola had many more taxa that were either positively or negatively correlated with rain, while bean and soybean had more taxa RA correlated with sampling date or developmental stage of the host (Supplementary Fig. S2). 
A

Low abundance genera
- WYO
"WS3
" TM6
- Verrucomicrobia
- Thermi
- Spirochaetes
- Proteobacteria
- Plantomycetes
- Nitrospirae
- Gemmatimonadetes
- Fusobacterium
- Firmicutes
- Chloroflexi
"Bacteroidetes
-Armatimonadetes
-Actinobacteria
-Acidobacteria
- Other

Date

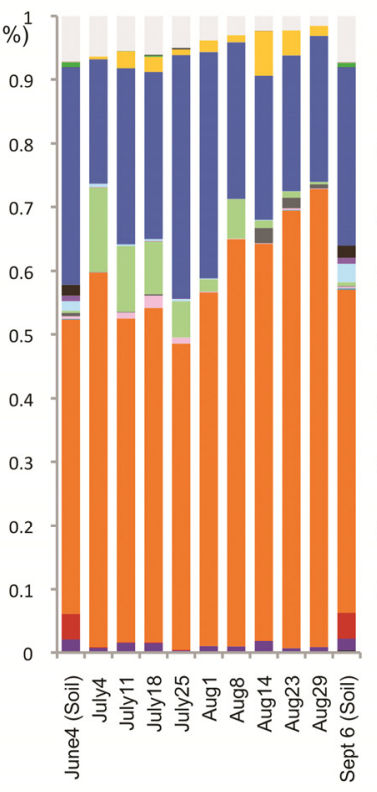

Development
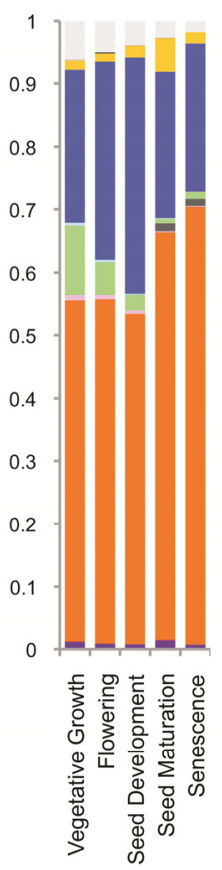

Plot

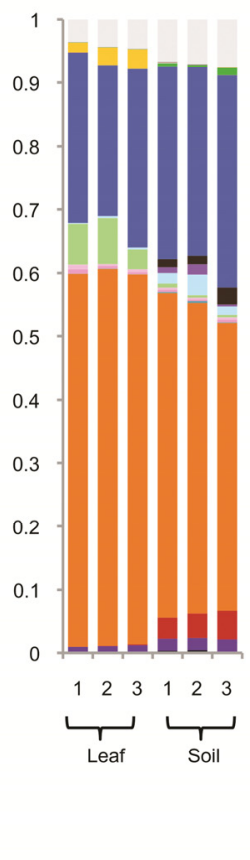

Species

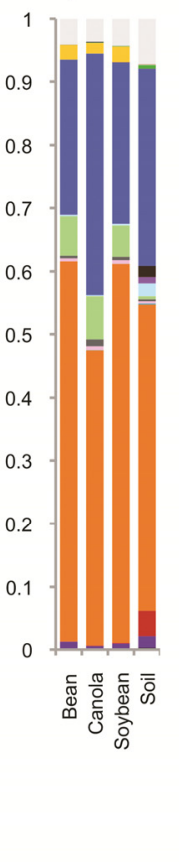

Cultivar

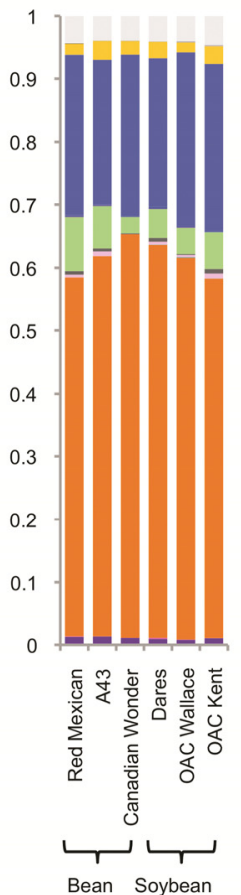

B

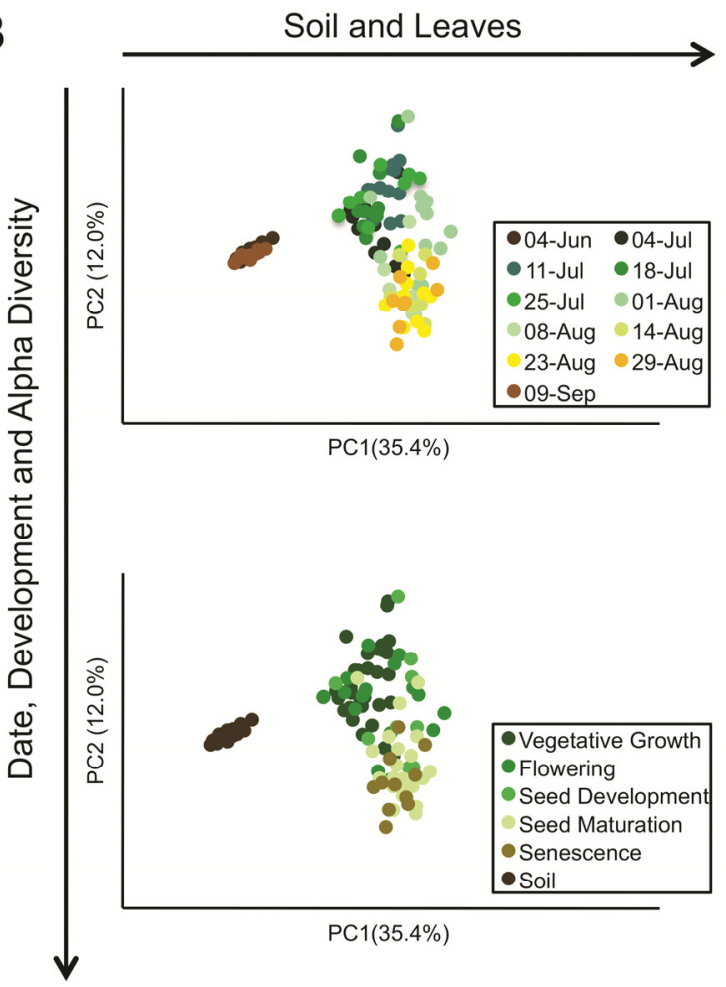

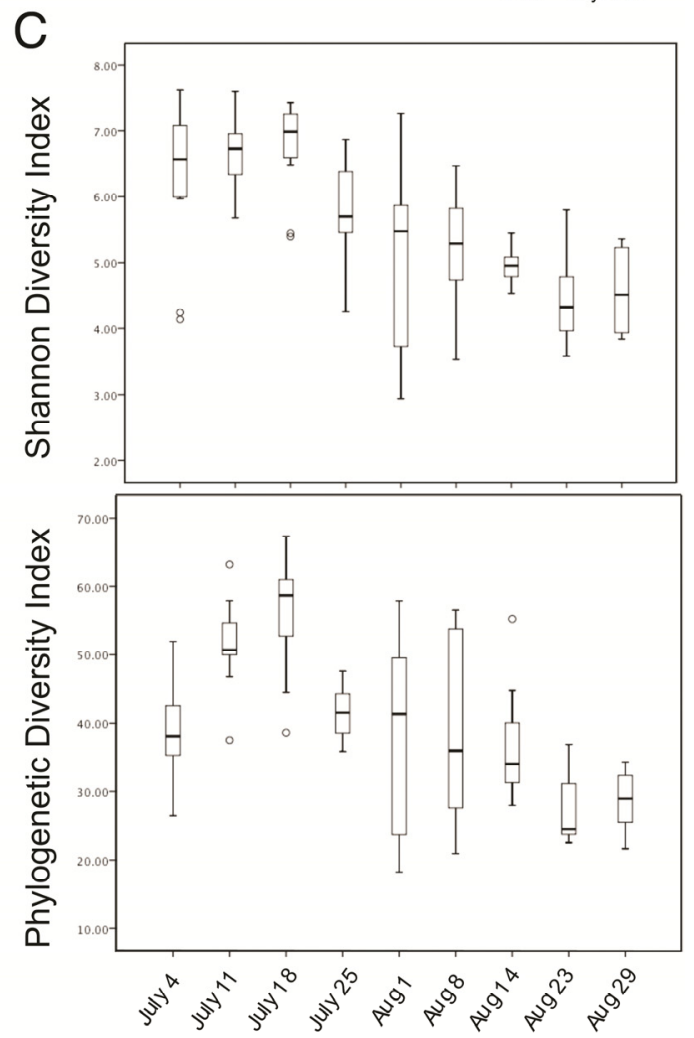

Sampling Date

Fig. 1. Community structure and diversity is determined by sampling date and developmental stage of the host. A, Relative abundance (RA) of phyla present in the soil and leaf microbiome is displayed by sampling date, developmental stage of the plant, plot location, host species, and host cultivar. Nonparametric analysis of variance indicates that the distribution of phyla was significantly different $(P<0.05$, Bonferroni corrected $)$ between soil and leaf samples, over sampling dates, and as the plant develops. Plot location, host species or any of their respective cultivars did not show significant differences in the distribution of phyla. B, Principal coordinates analysis (PCoA) based on weighted UniFrac distances and coded for sample collection date or plant developmental stage. PC1, which explains $35.4 \%$ of variance, separates soil and leaf samples, while PC2, which explains $12 \%$ of variance, separates leaf samples by date, developmental stage of the host, and $\alpha$ diversity. $\mathbf{C}$, Shannon and phylogenetic diversity significantly $(P<0.005)$ decreases in the leaf samples over the course of the growing season. 
A distinct community of leaf-specific bacteria develops.

The majority of bacteria were shared between the soil and leaf communities; however, there were also many genera present in the leaf tissues that were not found in the soil. These bacteria were potentially from other sources such as rain, wind, neighboring vegetation, or the seed. By eliminating the genera identified in the soil microbiome, we can examine the community members that are found exclusively in the leaves (i.e., the leaf-specific microbiome). The PCoA plots of weighted UniFrac distances determined at the OTU level for the whole community revealed that, as time progressed, there was a significant increase in weighted UniFrac distance be- tween the leaf samples and the soil samples $(P<0.005)$ (Fig. $3 \mathrm{~A})$. This continuing separation of the leaf community from the soil community suggests that the establishment and proliferation of a leaf-adapted community, unique from the soil community, had occurred. There is also a significant increase $(P<0.005)$ in the proportion of leaf-specific genera over time. From the initial leaf samples taken on 4 July 2012, an average of $24.0 \%$ of the community was composed of genera found only in leaf samples (Fig. 3B). As the weekly samplings continued, the proportion of leaf-specific genera significantly increased, with the highest proportion of leaf-specific genera isolated on 23 August 2012 at an RA of $40.1 \%$.

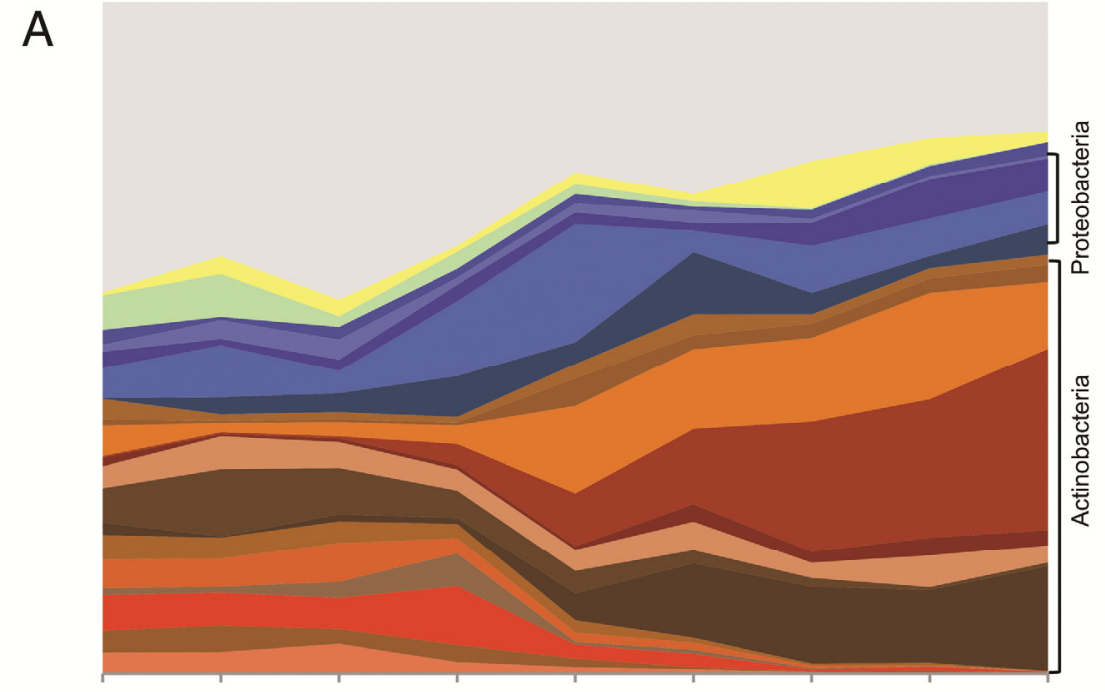

July4 July11 July18 July25 Aug1 Aug8 Aug14 Aug23 Aug29
Low Abundance (<1\%)

Deinococcus(Deinococcales)

Exiguobacterium(Bacillales)

- Pseudomonas(Pseudomonadales)

- Enterobacteriaceae, Other(Enterobacteriales)

- Comamonadaceae, Other(Burkholderiales)

- Oxalobacteraceae,Other(Burkholderiales)

- Sphingomonas(Sphingomonadales)

- Microbacteriaceae,Other(Actinomycetales)

- Microbacterium(Actinomycetales)

Frigoribacterium(Actinomycetales)

- Kineosporiaceae,Other(Actinomycetales)

- Kineococcus(Actinomycetales)

- Nocardioidaceae,Other(Actinomycetales)

- Geodermatophilaceae,Other(Actinomycetales)

- Geodermatophilus(Actinomycetales)

- Intrasporangiaceae,Other(Actinomycetales)

- Solirubrobacterales, Other(Actinomycetales)

- Pseudonocardiaceae,Other(Actinomycetales)

- Micrococcaceae,Other(Actinomycetales)

- Sporichthyaceae,Other(Actinomycetales)

- Gaiellales,Other(Actinomycetales)
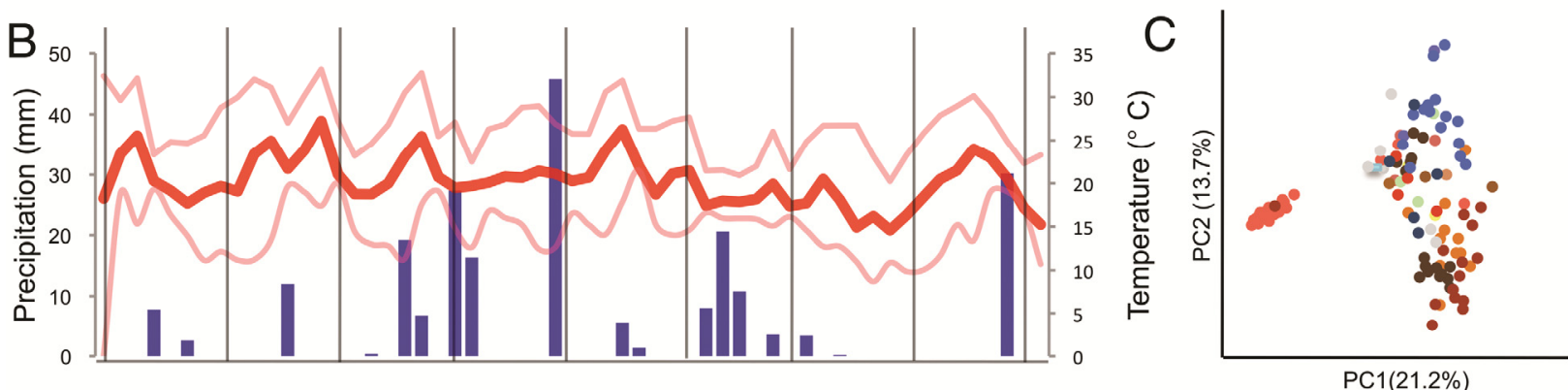

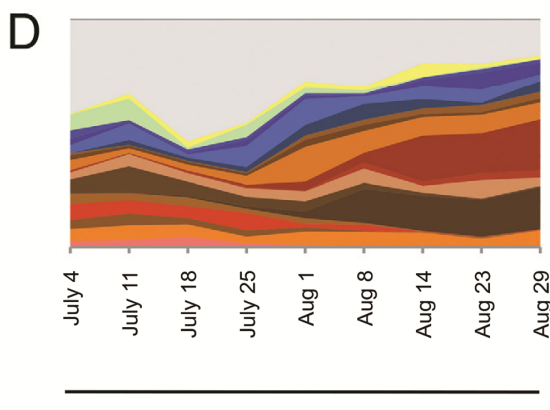

Bean

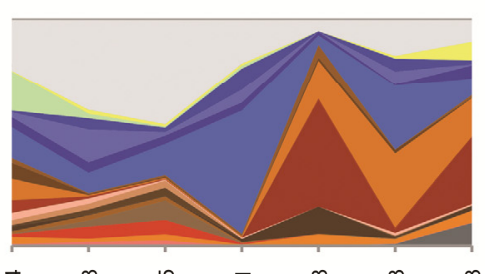

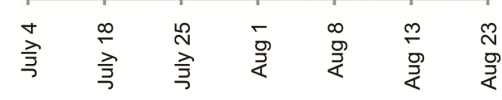

Canola
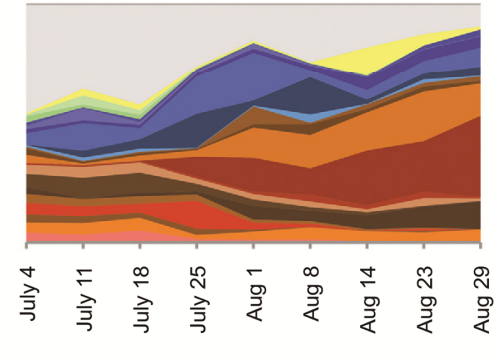

Soybean

Fig. 2. Succession of high-abundance genera throughout the season. A, Percent relative abundance (RA) for high-abundance genera are shown over the course of the growing season. An "Other" designation in the taxa list indicates that a genus could not be successfully identified for that operational taxonomic unit sequence. B, Daily temperature (daily average $=$ red line, low and high range $=$ pink lines) and precipitation (blue bars) over the course of the growing season. Temperature decreases and large rainfall events were experienced from 25 to 31 July 25 , with the largest volume of rainfall recorded all season $(45.8 \mathrm{~mm})$ on 31 July. These dates correspond to an increase in the RA of genera from the Actinobacteria. C, Principal coordinate analysis plot based on weighted UniFrac distances (as in Figure 1B) and coded by high-abundance taxa shows a separation among the samples primarily dominated by taxa from the order Burkholderiales to samples dominated by taxa from the order Actinomycetales. D, RA plots over time for each host species, showing that the shift in community structure occurs first in soybean, prior to the incidents of rainfall observed from 25 to $31 \mathrm{July} 25$, and before the appearance of mature seeds. 
At the phylum level, Actinobacteria, Firmicutes, Proteobacteria, and Thermi dominated the leaf-specific microbiome (Supplementary Fig. S3). The RA of these leaf-specific phyla and genera followed patterns similar to those of the whole leaf microbiome. A multiple linear regression found a significant decrease in PD $(P=0.000)$ but not in Shannon diversity $(P=$ 0.203 ) over time, resulting in sampling date being a significant predictor of PD $(P=0.000)$ but not Shannon diversity $(P=$ 0.054) (Supplementary Table S4). PD and Shannon diversity were both significantly lower for the leaf-specific microbiome than the whole leaf microbiome $(P<0.005)$.

PCoA of weighted UniFrac $\beta$ diversity determined for the leaf-specific microbiome shows a separation of samples by sampling date and developmental stage of the host on PC1 and by most abundant genus and plot location on PC2 (Fig. 4). Thus, these factors significantly $(P<0.05)$ predict the location of the sample on their respective influential axes (Supplementary Table S5). Host species and host cultivar did not separate by either axis.

For the whole microbiome, the average weighted UniFrac distance between all samples at a time point remained the same throughout the season $\left(R^{2}=0.038, P=0.464\right)$. For the leaf-specific microbiome, there was a significant decrease in weighted UniFrac distances between samples over the course of the sampling season $\left(R^{2}=0.857, P=0.000\right)$ (Fig. 3C). This suggests that, over time, the leaf-specific microbiome converged and became increasingly similar, with the same genera dominating the leaf microbiome, regardless of location or host species.

\section{Inference of community function.}

A phylogenetic investigation of communities by reconstruction of unobserved states (PICRUSt) imputation of microbiota function was determined in order to identify functionality of microbial communities, inferred from taxonomic identify. Significant differences $(P<0.05$, Bonferonni corrected for 4,450 tests) in the inferred metagenomic composition between the soil and the whole leaf microbiome were identified (Fig. 5). Pairwise comparisons of total clusters of orthologous group (COG) gene family counts between the soil microbiome and the leaf microbiome were performed per COG category. The leaf microbiome was significantly enriched in COGs categorized as carbohydrate transport and metabolism and nucleotide transport and metabolism. The COG gene categories significantly enriched in the soil microbiome were cell wall, cell membrane, and envelope biogenesis; energy production and conversion; and cell motility. The COG gene families and the RA of genera in both the whole microbiome and the leafspecific microbiome of bean and soybean are more similar to each other than to the gene families and genera RA present in canola (Supplementary Fig. S4). The branch lengths of the hierarchical clustering in both analyses show that the whole leaf microbiome is more similar between host species than the leaf-specific microbiome.
Symbiotic relationships of host-adapted taxa.

Network analysis was used to examine the relationship of genera abundances in the leaf microbiome. The analysis of positive taxa correlations clusters the microbiome into two distinct, highly connected clusters (Fig. 6). Cluster 1 can be further analyzed as a two-component cluster. One component consists of taxa always found at low RA, often found in soil samples, and rarely found in leaf samples, while the other component of cluster 1 consists of taxa that were moderately prevalent throughout leaf and soil samples and have extremely variable RA. Cluster 2 consists of many taxa that had a high average RA and were highly prevalent in the leaf, many increasing in RA over time, likely able to proliferate and adapt to living in the phyllosphere. Cluster 2 also consists of several low-abundant but highly prevalent taxa. These taxa were also likely host adapted and critical to the leaf microbiome because of their relationship with other host-adapted but highly abundant taxa. Leaf-specific genera make up $44 \%$ of cluster 2 but only $12 \%$ of cluster 1 . Potential plant pathogens from the genera Erwinia and Pseudomonas are found in cluster 1 and are closely clustered in the network. Other potential plant pathogens identified in our network analysis include Rathayibacter, Curtobacterium, and Sphingomonas spp. (Bull et al. 2010). These potential pathogens were all identified in cluster 2 and located in close proximity on one side of the network but are not all significantly correlated with one another.

Taxa identified in our analysis that could function as potential plant-growth-promoting bacteria (PGPB) include Exiguobacterium, Sphingomonas, Pseudomonas, Bacillus, and Methylobacteraceae (Berg et al. 2005; Innerebner et al. 2011; Madhaiyan et al. 2006; Rajendran et al. 2012). Exiguobacterium and Bacillus are located in cluster 1 while Sphingomonas, Pseudomonas, and Methylobacteraceae are located in cluster 2. As was the case for the cluster 2 pathogens, most of these cluster 2 potential growth promoters are not significantly correlated but are all located in close proximity on one side of the network. Interestingly, these potential growth promoters cluster on the opposite side of the network from the potential pathogens.

\section{DISCUSSION}

Identifying members of the phyllosphere microbiome and determining the forces that influence colonization, abundance, and prevalence in the phyllosphere is a relatively new field of study. Previous studies have attempted to determine the forces that significantly influence the leaf microbiome. Some of these studies suggest that geographical location has the greatest influence on community composition (Finkel et al. 2011; Rastogi et al. 2013; Redford et al. 2010), while other studies suggest that host genotype is the primary factor driving the community (de Oliveira Costa et al. 2012; Kim et al. 2012). Some studies also credit the soil and airborne microbiomes as having a significant influence on the phyllosphere microbiome

Table 1. Correlation between relative abundance and time for high abundance taxa ${ }^{\mathrm{a}}$

\begin{tabular}{ll}
\hline Direction of change $\mathbf{b}^{\mathbf{b}}$ & \multicolumn{1}{c}{ Taxonomy } \\
\hline Decreasing & Actinobacteria; Thermoleophilia; Gaiellales; Gaiellaceae; Unknown Genus \\
Decreasing & Actinobacteria; Actinobacteria; Actinomycetales; Sporichthyaceae; Unknown Genus \\
Decreasing & Actinobacteria; Actinobacteria; Actinomycetales; Intrasporangiaceae; Unknown Genus \\
Decreasing & Actinobacteria; Actinobacteria; Actinomycetales; Geodermatophilaceae; Unknown Genus \\
Decreasing & Firmicutes; Bacilli; Bacillales; Bacillales Family XII. Incertae Sedis; Exiguobacterium \\
Increasing & Actinobacteria; Actinobacteria; Actinomycetales; Microbacteriaceae; Frigoribacterium \\
Increasing & Actinobacteria; Actinobacteria; Actinomycetales; Geodermatophilaceae; Geodermatophilus \\
Increasing & Actinobacteria; Actinobacteria; Actinomycetales; Kineosporiaceae; Unknown Genus \\
\hline
\end{tabular}

\footnotetext{
${ }^{a}$ Relative abundance $>1 \%$.

b Taxa relative abundance versus time with a positive Pearson correlation value $>0.8$ or a negative Pearson correlation value $<-0.8$.
} 
(Bodenhausen et al. 2013; Hardoim et al. 2011; Maignien et al. 2014). Despite this advancement in our understanding of the phyllosphere community, much of the variation observed in the phyllosphere remains unexplained, suggesting that other factors may be playing a significant role in determining community composition. Recent studies have begun to investigate the effect of migration and community succession in the phyllosphere microbiome, suggesting that colonization, persistence, and succession of the community may be a key factor driving the phyllosphere microbiome (Maignien et al. 2014; Redford and Fierer 2009; Shade et al. 2013). Our study considered the effect of biological and environmental conditions such as host genotype and weather events while monitoring changes over time to identify the primary factors that determine the composition of the phyllosphere microbiome. Our results show that the composition of the community is primarily driven by temporal changes and community succession, whereas host species and geographical location do not have a strong impact on the community.

Geographical location appeared to have very little effect on community composition. Previously, location-dependent differences observed in the soil or leaf microbiome were between samples taken from distant locations with very different climates and soil properties (Badri et al. 2013; Finkel et al. 2011; Peiffer et al. 2013; Redford et al. 2010). Our locations are all within $2 \mathrm{~km}$ of each other, and the similarity among soil microbiomes suggests that the plots have similar ecological properties. When the leaf-specific microbiome was examined, community differences between plot locations were observed at the beginning of the sampling season. Leaf-specific communities then converged over time and, by the end of the sampling season, plot location did not have a significant impact on community composition (Fig. 4E). This convergence of leaf-specific taxa over time and the lack of plot specificity for the whole microbiome is likely also a result of microbiota transferred between plants by wind or contamination during repeated samplings.

Although phyllosphere microbial community composition has been previously shown to be influenced by host species and genotype (Bálint et al. 2013; de Oliveira Costa et al. 2012; Kim et al. 2012; Knief et al. 2010), we did not see a strong host-specific signal. The interaction between the leaf and soil microbiomes, as well as the forces that drive microbiome succession over time, appears to have overwhelmed the host-spe-
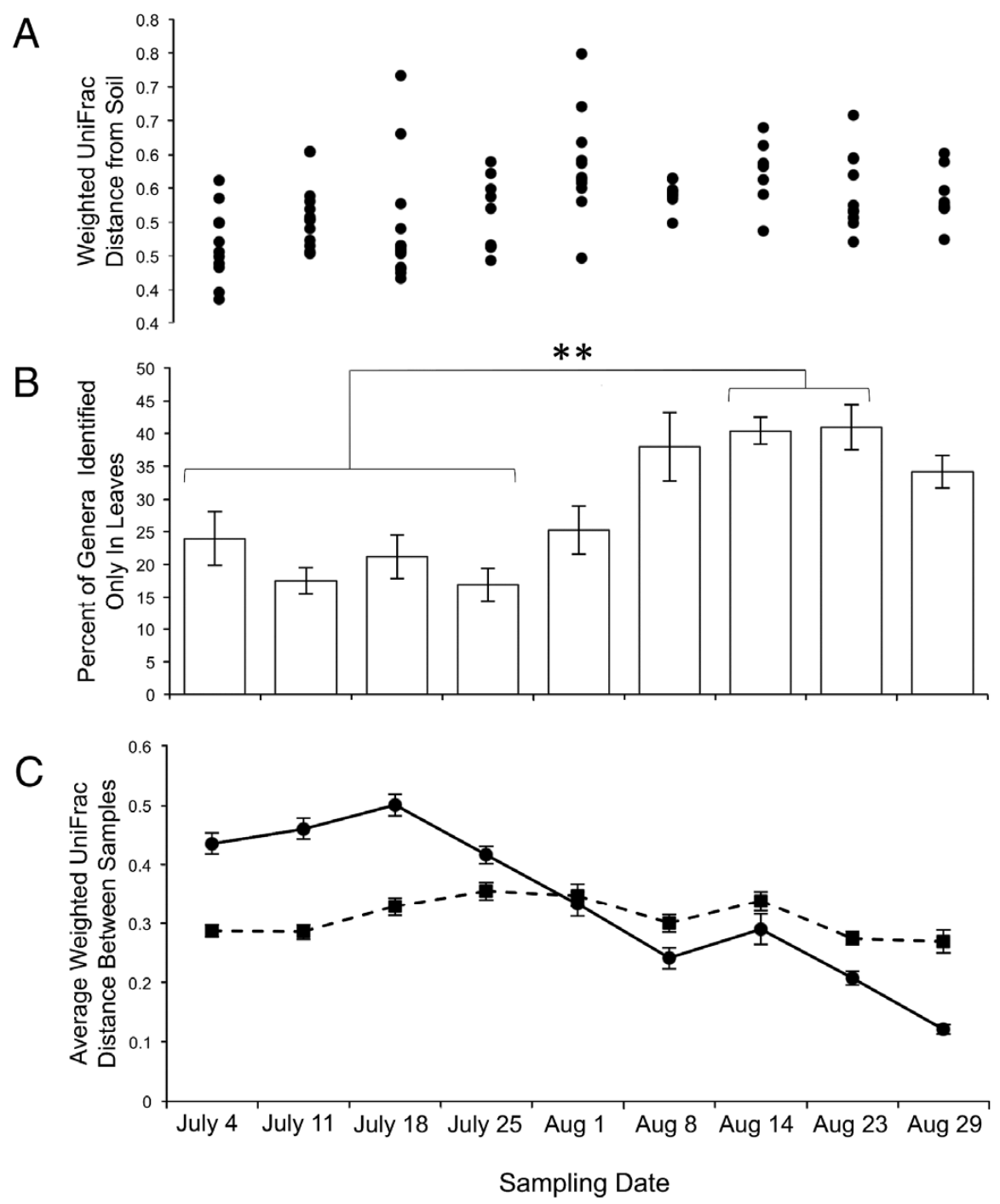

Fig. 3. Development and convergence of the leaf-specific microbiome. A, Average weighted UniFrac distance between leaf samples and soil samples increases over the course of the summer. B, Percent genera from each sample found only in leaves (mean \pm standard error) over the course of the growing season. There is a significant (** indicates $P<0.005$, Tukey post-hoc) increase in the relative proportion of leaf-specific genera in samples collected later in the growing season. C, Weighted UniFrac distances between all samples at a given time point for the leaf-specific microbiome significantly decrease over the course of the growing season (solid line), while the same measure for the whole microbiome remains relatively constant (dashed line). 
cific signal in our case. These different conclusions may be due to a number of reasons. In the case of phyllosphere microbiomes from trees or perennial species, there may be less migration from the soil simply due to distance as well as a longer time for host adaption (Kim et al. 2012). The host specificity observed previously in studies of bean and Arabidopsis thaliana may be partially due to the use of culture-dependent techniques, the investigation of only subsets of the community (e.g., endophytes from the bean and Methylobacterium from the $A$. thaliana), or host species under investigation.

Though there is no strong host association observed through PCoA or RA at the phylum taxonomic level, clustering of the RA at the genus level and among COG gene families from the functional analysis using PICRUSt shows that the bean and soybean leaf microbiomes are more similar to each other than to the canola leaf microbiome. Bean and soybean leaves are similar in that they are both flat and broad and grow in trifoli- ate arrangements. Soybean leaves differ from bean leaves because they are covered in pubescence, or fine hair-like structures, which likely affects bacterial colonization (Gunasinghe et al. 1988; Johnson 1975). Nonetheless, bean and soybean are both from the family Fabaceae and, therefore, share many similar characteristics. Canola is from the family Brassicaceae, and the leaves grow in a rosette formation until the main stem forms, after which leaves are produced along the stem of the canola plant as well (Allen et al. 1971; Bouttier and Morgan 1992). These structural differences result in differences in proximity to the soil, leaf angle, leaf size, and leaf texture, which is reflected by the differences in genera RA and functional profile.

The PICRUSt analysis also revealed a significant enrichment of carbohydrate transport and metabolism and nucleotide transport and metabolism in the leaf microbiome compared with the soil microbiome, which likely reflects the nutrient

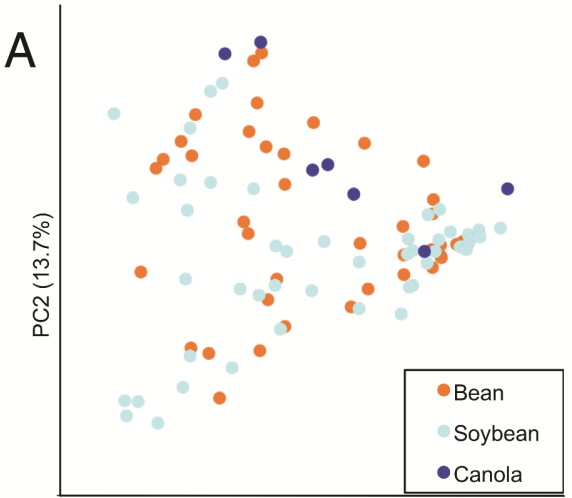

PC1 $(21.2 \%)$
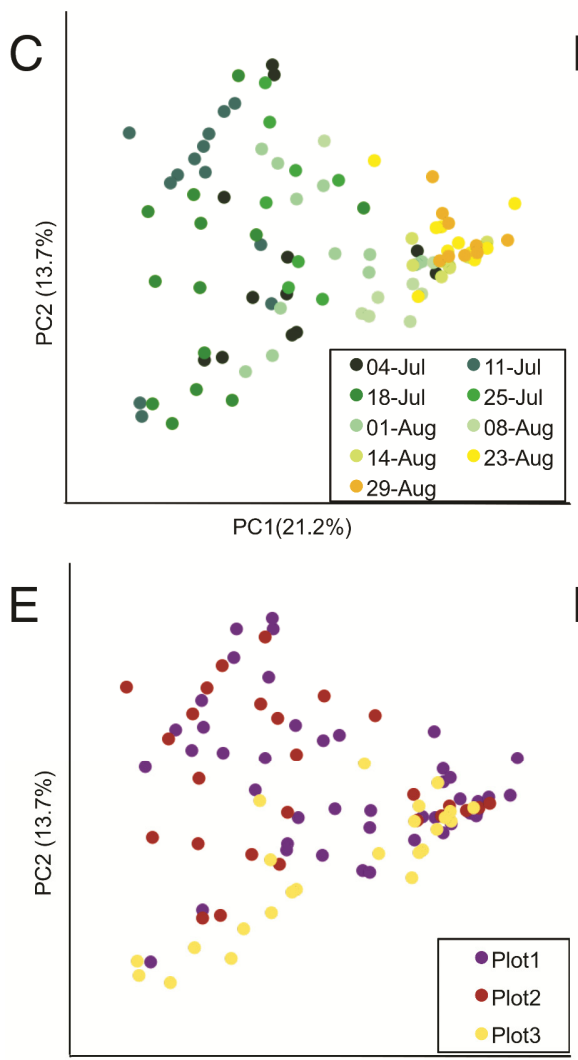

PC1 $(21.2 \%)$

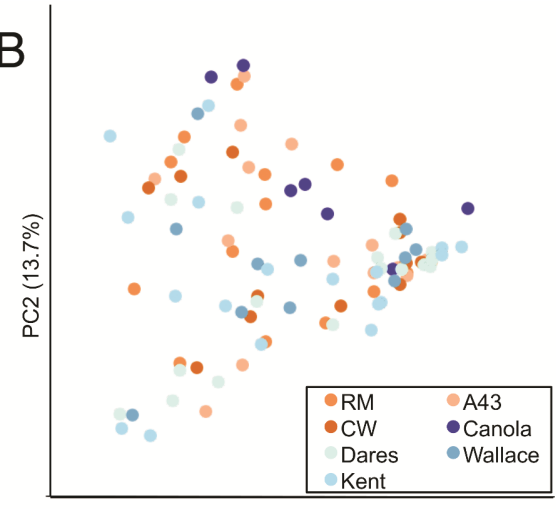

PC1 $(21.2 \%)$
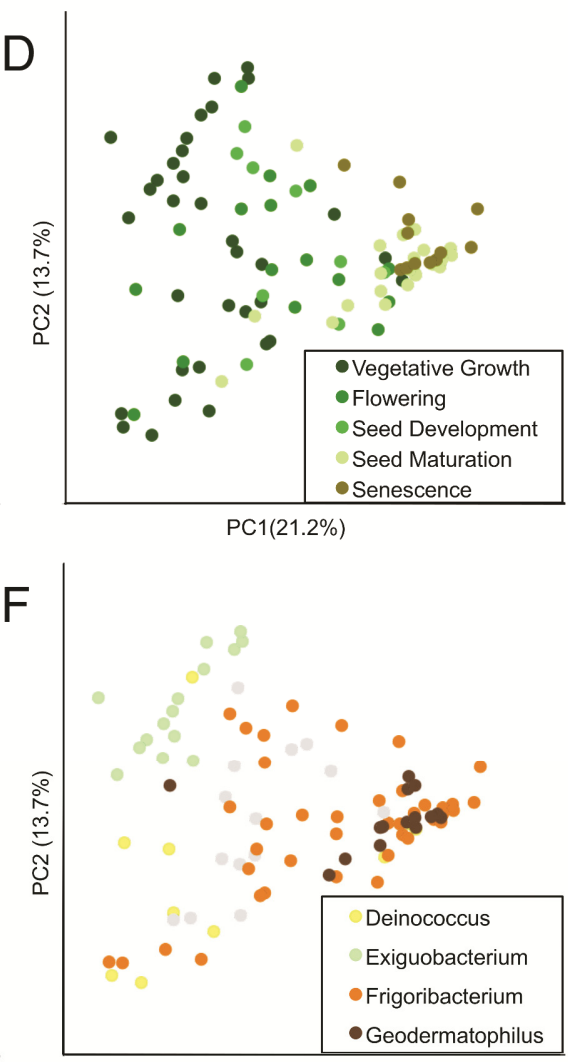

$\operatorname{PC} 1(21.2 \%)$

Fig. 4. Principal coordinate analysis (PCoA) of leaf-specific weighted UniFrac $\beta$ diversity. PCoA plots are colored by A, host species; B, host cultivar; C, sampling date; D, developmental stage of the plant; $\mathbf{E}$, plot location; and $\mathbf{F}$, most abundance genera. Samples separate by sampling date, developmental stage, plot location, and most abundant genera but not by host species or host cultivar. 


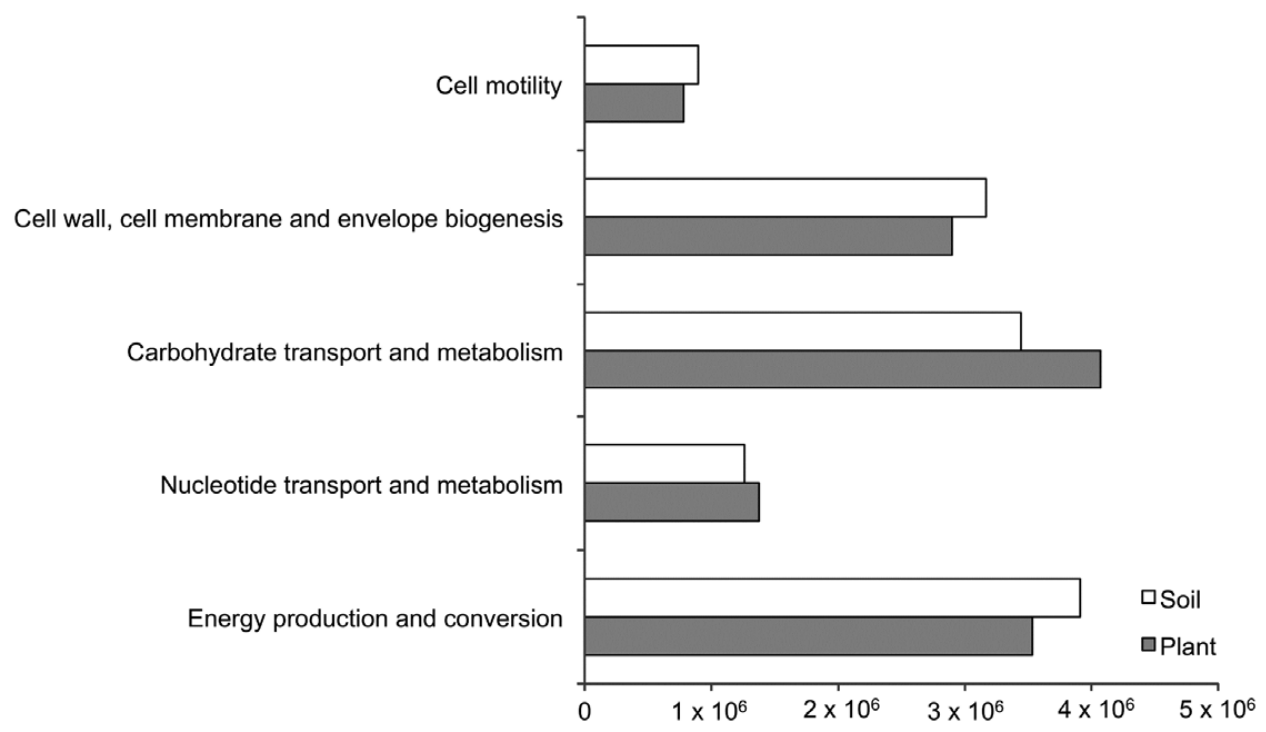

Fig. 5. Total gene families observed in the soil and the leaf microbiome. Total counts of gene families per clusters of orthologous group category that differ significantly $(P<0.05$, Bonferonni corrected for 4,450 tests) between the soil and leaf microbiome are shown, as determined by a PICRUSt imputation of microbiota function.

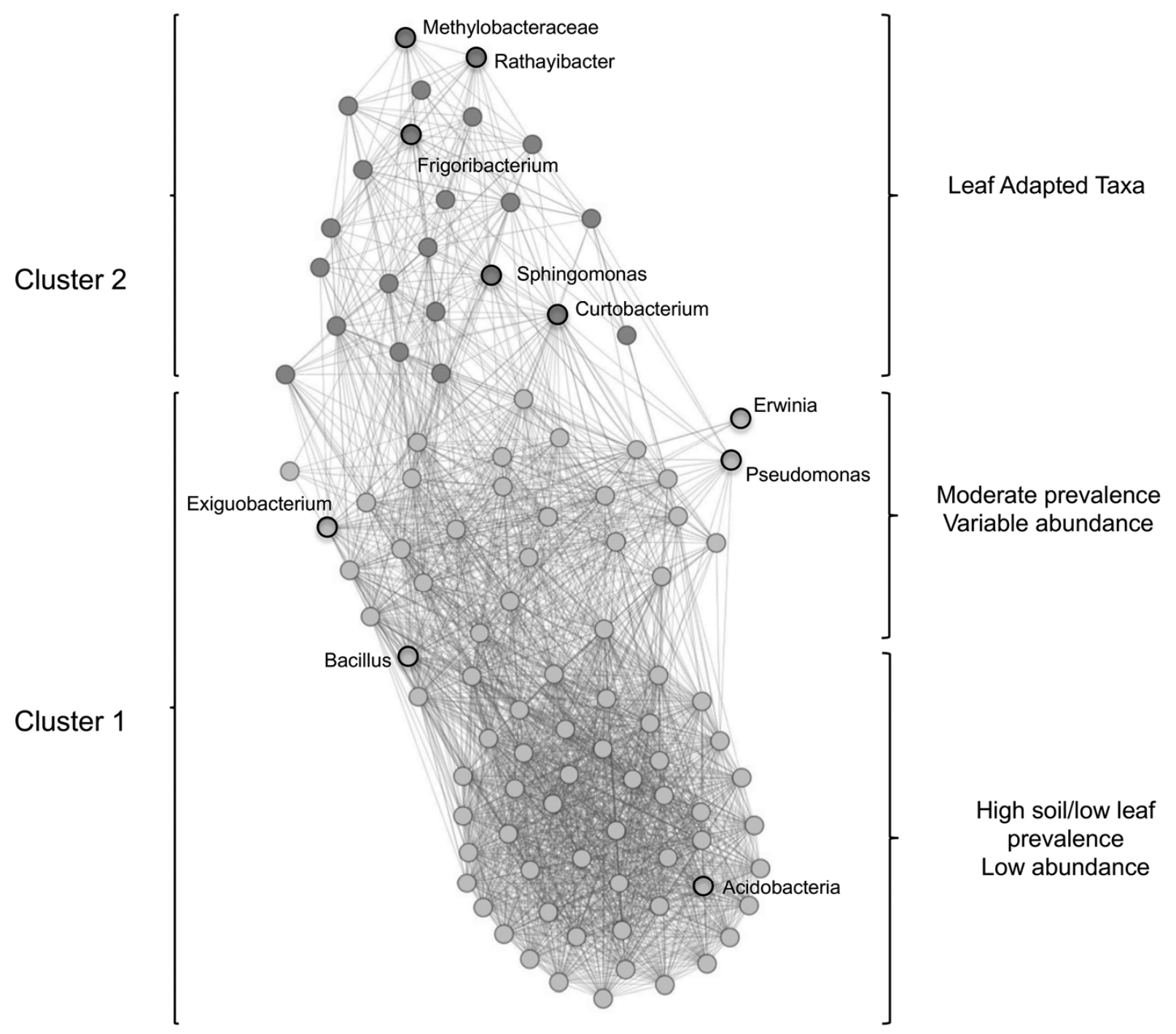

Fig. 6. Network analysis reveals trends in plant-adapted and beneficial bacteria. A Spearman correlation network was determined for taxa with an average relative abundance $>0.1 \%$. Network analysis determined that two main clusters of highly correlated taxa are formed. Cluster one contains taxa present in soil and leaves, with varying prevalence and abundance. Cluster 2 contains many taxa that are highly prevalent in leaf tissues, many increasing in relative abundance over time. 
availability and growth conditions of the leaf environment. This is compared with cell motility; cell wall, cell membrane, and envelope biogenesis; and energy production and conversion, which are significantly reduced in the leaf microbiome relative to the soil microbiome. The enrichment and reduction of these gene families in the leaf microbiome reflects the nutrient status of the phyllosphere. Taxa that are able to utilize a wide variety or specific subset of carbohydrates will flourish in the phyllosphere, as reflected in the enrichment for carbohydrate transport and metabolism gene families. Conversely, the high carbohydrate availability may decrease the importance of nutrient foraging and, therefore, the relative frequency of motility-associated genes. Alternatively, some motility mechanisms (e.g., the bacterial flagella) actually elicit host immunity and, therefore, taxa containing these mechanisms may be selected against in this environment (Gomez-Gomez et al. 1999)

Community succession was observed throughout the sampling season because the richness, evenness, and diversity decreased from the beginning of the sampling season to the end. At the beginning of the summer, the phyllosphere microbiome was very diverse and clustered more closely with the soil microbiome. As the summer continued, the evenness and richness significantly and predictably decreased. This trend was observed regardless of whether we considered simply the OTU present (Supplementary Fig. S5) or the RA of these species (Fig. 1). The proportion of leaf-specific bacteria significantly increased from July to August, contributing largely to the shift away from the soil microbiome. Specific rainfall events may also have acted as catalysts to community succession. These results contrast with those found by Redford and Fierer (2009), who did not show any predictable changes in diversity on cottonwood trees throughout the growing season, and Maignien and associates (2014), who showed a predictable increase in the diversity of the A. thaliana phyllosphere. Notably, these $A$. thaliana plants were grown in sterile media, in a sterile environment, and were only exposed to airborne bacteria as colonizers. Because the observed diversity steadily increased in the phyllosphere of these plants, it suggests that either a climax community was never achieved or that the persistence of dead cells or extracellular DNA resulted in inflated diversity. In the former case, a higher input of soil or airborne bacteria may be required to reach a stable equilibrium in the phyllosphere. The increase in phylogenetic diversity we observed between 4 and 18 July 2012 was likely the result of the soil and air continually inoculating the leaves with a range of taxa. Once enough successful colonizers and leaf-adapted taxa are present on the leaf, they may begin to out-compete other taxa, reducing the overall diversity observed on the leaf, as we saw following 18 July 2012.

Our network analysis reveals an otherwise unidentifiable relationship among phyllosphere bacteria. Persistent taxa have correlated patterns of RA that cause them to cluster in one region of the network, while fluctuating, low-abundant, and transient bacteria form a second cluster. Low-abundant yet persistent taxa may be particularly interesting because their persistence suggests that they are well adapted to the leaf environment, and their high network connectivity indicates that they may be keystone species in the phyllosphere. Network analysis also reveals patterns that separate potential PGPB and potential pathogens, although these conclusions are made challenging due to the difficulty of reliably resolving taxonomy below the genus level. Nevertheless, it is intriguing that potential pathogens such as Erwinia and Pseudomonas cluster on one side of the network while potential growth promoters such as Exiguobacterium and Bacillus cluster together but on the opposite side of the network. Consequently, taxa that are positively correlated with known growth promoters and negatively correlated with potential pathogens may be good candidates for future biocontrol agents or PGPB. In our data, taxa that are correlated with potential growth promoters include Terracoccus, Deinococcus, or Caulobacter. Methylobacteraceae is also distantly located from many of these potential pathogens and is in the same bacterial order as Rhizobium spp., which have shown to be effective PGPB in the rhizosphere (Compant et al. 2005). Many of these bacteria have otherwise never been tested as PGPB but, based on their proximity to other potential growth promoters, may be likely candidates for testing. Understanding the symbiotic relationship between these hostadapted microbes and the role they plant in plant health and development may be key to successfully developing synthetic leaf communities for biocontrol (Andrews 1992).

In conclusion, this study shows that, in a natural environment, the phyllosphere microbiome of seasonal agricultural crop plants is primarily influenced by temporal community succession. Although we find little role for host genotype and spatial distribution, this may simply reflect the specific choice of study species and the very small geographic distances between our replicate plots. Communities sampled at the beginning of the summer more closely resembled the soil community and, as the season progressed, the phyllosphere microbiome became increasingly distinct and less diverse. Consequently, taxa that may be useful biocontrol agents or PGPB in the early summer may not be as effective in late summer because of the dramatic shift in community composition and the decrease in diversity. By analyzing highly correlated taxa we can determine those that are essential to the leaf microbiome, and potentially identify previously unidentified taxa that may play a role in plant protection and growth promotion. However, it is also essential to understand that the natural succession of the community is driving the structure of the microbiome and that successful biocontrol in agricultural crops will require knowing how these changes shape the community over the course of the growing season.

\section{MATERIALS AND METHODS}

\section{Study design and sample collection.}

Plants were grown at the University of Toronto Koffler Scientific Reserve (KSR) in King, Ontario, Canada. G. max 'OAC Wallace', 'OAC Kent', and 'Dares'; $B$. napus 'Westar'; and $P$. vulgaris 'Red Mexican', 'Canadian Wonder', and 'A43' seed were planted at three locations at the KSR on 4 June 2012. These cultivars of soybean and canola are all commonly farmed in Ontario and have been bred to grow ideally in the shorter Canadian growing season and temperate climate (Mahuku et al. 1997; Zhang et al. 2010). The bean cultivars differ in the presence or absence of resistance $(R)$ genes specific to certain strains of Pseudomonas syringae pv. phaseolicola. The three locations (plot 1: $44^{\circ} 1^{\prime} 42.97^{\prime \prime} \mathrm{N}, 79^{\circ} 32^{\prime} 6.39^{\prime \prime}$ $\mathrm{W}$; plot 2: $44^{\circ} 1^{\prime} 47.18^{\prime \prime} \mathrm{N}, 79^{\circ} 32^{\prime} 8.53^{\prime \prime} \mathrm{W}$; and plot 3 : $44^{\circ} 1^{\prime} 29.99^{\prime \prime} \mathrm{N}, 79^{\circ} 32^{\prime} 10.76^{\prime \prime} \mathrm{W}$ ) differed by observable surrounding vegetation, exposure to environmental conditions, and land history. Within each plot, 16 replicate rows were planted. The location of each plant species in each row and the order that the rows were harvested was determined randomly but was identical between each plot.

Starting 4 July 2012, one leaf from every plant within a row was collected weekly. Sampling continued until 29 August 2012, when most plants showed signs of senescence. Six soil samples from each plot were collected prior to planting on 4 June 2012 and following final leaf collection on 6 September 2012. Leaf samplings occurred between 9:00 a.m. and 12:00 p.m. Leaves were collected from plants within the same row. If a plant was missing from that row due to disease, severe her- 
bivory, or failure to germinate, that plant species was omitted from sampling at that time. Stage of plant development was noted as either vegetative growth, flowering, seed development, seed maturation, or senescence (Supplementary Fig. S6). Leaves were removed from each plant using ethanol-sterilized forceps and immediately collected in a sterile Ziploc plastic bag. Leaves were collected from the upper canopy of the bean and soybean and from the canola rosette, as well as the stem as the canola matured. Leaves were transported to the University of Toronto, St. George campus, within 2 to $4 \mathrm{~h}$ of sampling. Four cores were then taken from each leaf using a sterile cork borer (3/8 in. diameter), allowing a range of areas on the leaf to be sampled while not exceeding the desired leaf weight for storage and small-volume DNA extraction. Leaves were not surface sterilized to ensure that both endophytic and epiphytic communities could be recovered. Excess dirt was shaken off during the coring process. Leaf cores were frozen using liquid nitrogen and stored in DNA-free, RNA-free sterile $1.7-\mathrm{ml}$ centrifuge tubes at $-80^{\circ} \mathrm{C}$. Soil samples were collected at the depth of seeding ( 1 to 2 in. below the surface) in 50-ml sterile Falcon tubes and stored at $4^{\circ} \mathrm{C}$ until DNA extraction. The remaining soil was distributed into $1.7-\mathrm{ml}$ sterile centrifuge tubes and frozen at $-20^{\circ} \mathrm{C}$.

\section{Environmental measurements.}

Temperature and relative humidity measurements were collected on-site every 15 min using the HC-S3 Rotronic HygroClip Probe (Campbell Scientific, Edmonton, Canada) Daily precipitation measurements were recorded at the Buttonville weather station $\left(43^{\circ} 51^{\prime} 44.00^{\prime \prime} \mathrm{N}, 79^{\circ} 22^{\prime} 12.00^{\prime \prime} \mathrm{W}\right)$, roughly $22.9 \mathrm{~km}$ from the research station, and accessed through Environment Canada (Government of Canada). Total daily and weekly precipitation was determined using data until 1 day prior to sampling because samplings occurred during the mornings and data were not available hourly.

\section{Extraction and amplification of microbial DNA from soil and leaf tissues.}

Genomic DNA was extracted from leaf samples using the Wizard genomic DNA purification kit (Promega Corp., WI, U.S.A.), following the plant DNA extraction protocol as specified by the manufacturer, with the addition of lysozyme prior to cell lysis and increased lysis temperature, as described in the bacterial DNA extraction protocol. Genomic DNA was extracted from soil samples using the E.Z.N.A Soil DNA kit (Omega Bio-Tek Inc., GA, U.S.A.). To minimize the effect of PCR conditions on community amplification, samples were processed in a random order. The V5, V6, and V7 hypervariable regions of the $16 \mathrm{~S}$ rRNA gene were amplified using the 799f/1193r primers and the touch-down PCR method established by Bugarelli and associates (2012) (Chelius and Triplett 2001). A 25- $\mu$ l PCR reaction consisted of $1 \times$ KAPA2G Robust HotStart ReadyMix, $0.6 \mu \mathrm{M}$ each primer, and between 1 and 4 $\mu \mathrm{l}$ of DNA template. For every PCR reaction, sterile distilled (d) $\mathrm{H}_{2} \mathrm{O}$ was used as a negative control to ensure that no contaminating bacterial DNA was present, and $P$. aeruginosa $\mathrm{PAO} 1$ was used as a positive control for bacterial amplification. If inadequate amplification was observed following the PCR reaction to carry through, the PCR was repeated. Depending on the success of the reactions, either the duplicates were pooled or only the new reaction was used. Following amplification, the PCR product was used as a template for a secondary PCR using the V5+791 and V7-1104 primers with 8-mer barcodes on the $5^{\prime}$ ends for multiplexing (Maughan et al. 2012). Every 25- $\mu$ l PCR reaction for secondary PCR consisted of the same reagents as mentioned above. The $\mathrm{dH}_{2} \mathrm{O}$ negative control from the initial round of amplification was carried through the secondary PCR to ensure that the additional PCR cycling did not result in amplification of any bacterial DNA. Amplification conditions for the secondary PCR were $3 \mathrm{~min}$ at $95^{\circ} \mathrm{C}$; eight cycles of $15 \mathrm{~s}$ at $95^{\circ} \mathrm{C}, 15 \mathrm{~s}$ at $50^{\circ} \mathrm{C}$, and $15 \mathrm{~s}$ at $72^{\circ} \mathrm{C}$; followed by a 5 -min final extension at $72^{\circ} \mathrm{C}$. The same PCR reaction was performed with each primer set, sterile $\mathrm{dH}_{2} \mathrm{O}$, and 25 cycles of $15 \mathrm{~s}$ at $95^{\circ} \mathrm{C}, 15 \mathrm{~s}$ at $50^{\circ} \mathrm{C}$, and $15 \mathrm{~s}$ at $72^{\circ} \mathrm{C}$ to confirm that no bacterial DNA was present in any of the primers. The success of the amplification and the approximate quantity of amplified DNA was determined by running the samples on a $1 \%$ agarose gel and determining the band intensity of the 16S rRNA gene band. Barcoded samples were then pooled by approximately even concentrations and run on a $1 \%$ low melting temperature agarose gel. Amplicons were size selected to remove any amplified mitochondrial DNA using NucleoSping purification columns (Macherey-Nagel Inc., PA, U.S.A.). Four MiSeq sequencing runs were required to sequence all samples. $P$. aeruginosa PAO1 was run as a single-species positive control for each sequencing run (Maughan et al. 2012).

\section{Illumina paired-end MiSeq sequencing of the V5 to V7 hypervariable regions of the $16 \mathrm{~S}$ rRNA gene.}

Amplicon libraries were constructed following standard Illumina paired-end library sample preparation protocols, minus fragmentation. End repair, A-tailing, adaptor ligation, and amplification were performed with reagents from NEBNext DNA Library Prep Master Mix Set for Illumina (New England Biolabs, MA, U.S.A.). 16S rRNA gene sequencing of the V5 to V7 region was performed on an Illumina MiSeq with V2 (250 bp $\times$ two paired end reads) or V3 (300 bp $\times$ two paired end reads) chemistry (Illumina Inc., CA, U.S.A.). Samples were multiplexed up to 96 samples per sequencing run.

\section{Community analysis using Mothur and QIIME.}

Mothur v.1.31.0 was used for initial quality trimming and assembly of reads (Schloss and Handelsman 2006; Schloss et al. 2009). Sequence fragments with a quality average less than 25 or a quality minimum less than 20 , within a 50 -bp sliding window, were removed. Sequences were then grouped according to the barcode and primer. A $25 \%$ base pair misidentification errorrate was allowed for the barcodes and a $15 \%$ misidentification error rate was allowed for the primers. Read 1 and read 2 sequences were assembled to create one full-length V5, V6, and $\mathrm{V} 7$ contig. All reads in the reverse orientation (V7, V6, V5) were reverse complimented and both datasets were combined per sample. All sequences were screened to removed sequences with high runs of homopolymers $(>6)$ or outside the expected sequence length ( $<345$ and $>358 \mathrm{bp}$ ), determined by the software program TaxMan (Brandt et al. 2012).

Further analyses were performed using Quantitative Insights Into Microbial Ecology (QIIME), version 1.8.0. Chimeras were identified in the FASTA sequences using the usearch61 de novo method (Edgar 2010). The chimera-free FASTA sequences were clustered into OTU at $97 \%$ identity using the de novo clustering algorithm UCLUST (Edgar 2010). A representative sequence from each OTU was picked and aligned using PyNAST to the GreenGenes 16S rRNA bacterial database, version 13.5 (Caporaso et al. 2010; DeSantis et al. 2006; McDonald et al. 2012). Each representative sequence was also assigned a taxonomy using the Ribosomal Database Project Bayesian algorithm and the GreenGenes database, version 13.5 (Wang et al. 2007). Utilizing the taxonomic assignments and the alignment of the representative sequences, an OTU table was created. Sequences that failed to align to the reference database were eliminated from the table. Singletons and OTU with an RA of less than $0.005 \%$ were removed from the 
analysis (Bokulich et al. 2013). A phylogenetic tree of all sequence data were made from the alignment using FastTree (Price et al. 2009).

The data were rarefied to the number of sequences present in the lowest 10th percentile. Shannon diversity and PD indices were calculated using the rarefied OTU table. Weighted UniFrac was calculated to assess $\beta$ diversity (Lozupone and Knight 2005; Lozupone et al. 2011). An average of $99.903 \%$ of sequence reads for the single-species controls were successfully classified to the genus level. After clustering OTU by genus, any genus present in the soil or leaf samples with an RA of $<0.1 \%$ per sample was removed from the analysis.

\section{Statistical analysis.}

All statistical analyses were done using SPSS version 20.0.0. Linear regression analysis was used to identify relationships and determine Pearson correlation coefficients. Due to the nonparametric distribution of typical microbial communities, Mann-Whitney U and Kruskal-Wallis tests were used to determine significant changes in community composition between sampling dates, host, plot, and cultivar. Heatmaps were created using the Heatmap tool provided by the HIV Sequence Database.

\section{PICRUSt analysis.}

Using the $16 \mathrm{~S}$ rRNA gene data, the metagenome of the leaf and soil community was predicted using PICRUSt (Langille et al. 2013). PICRUSt analysis was performed on Galaxy, a Webbased platform for genomic analysis (Blankenberg et al. 2010; Giardine et al. 2005; Goecks et al. 2010). The COG database was selected and used to determine gene families (Tatusov et al. 2000).

\section{Network analysis.}

Spearman's rank correlation coefficient was used to calculate the correlations between genera. It has been previously shown that Spearman correlation coefficients can successfully detect true correlations within both gradient and clustered data (Kuczynski et al. 2010). Correlation values were calculated on the RA of genera with an average RA greater than $0.1 \%$. Swap randomization approach was used to permute the original RA data before recalculating the Spearman correlation for the genus (Gionis and Mannila 2007). This sequence of swap randomization and correlation recalculation was repeated 10,000 times. The observed correlations were then contrasted to the 0.99 quantiles of the distribution of random correlations to find whether the correlated RA data had a significantly higher correlation than random permutation. These significant correlations, representing significant associations between genera, were then used to build the microbial community network. The Markov clustering algorithm was used to find clusters of highly interacting genera within the microbial community network (Van Dongen 2000).

\section{ACKNOWLEDGMENTS}

We thank D. Desveaux for discussions and input and members of the Guttman and Desveaux Labs and the Centre for the Analysis of Genome Evolution and Function for support and discussion, A. Weis and J. Stinchcombe for their guidance and assistance at the Koffler Scientific Reserve, and S. Wadgymar for meteorological data from the Koffler Scientific Reserve. This work is supported by an NSERC Discovery Grant to D. Guttman. The authors declare no conflict of interest.

\section{LITERATURE CITED}

Allen, E. J., Morgan, D. G., and Ridgman, W. J. 1971. A physiological analysis of the growth of oilseed rape. J. Agric. Sci. Cambridge 77:339-341.
Andrews, J. H. 1992. Biological control in the phyllosphere. Annu. Rev. Phytopathol. 30:603-635.

Badri, D. V, Zolla, G., Bakker, M. G., Manter, D. K., and Vivanco, J. M. 2013. Potential impact of soil microbiomes on the leaf metabolome and on herbivore feeding behavior. New Phytol. 198:264-273.

Bálint, M., Tiffin, P., Hallstrom, B., B, O’Hara, R., Olson, M. S., Fankhauser, J. D., Piepenbring, M., and Schmitt, I. 2013. Host genotype shapes the foliar fungal microbiome of balsam poplar (Populus balsamifera). PLoS One 8:e53987. Published online.

Berg, G., Krechel, A., Ditz, M., Sikora, R. A., Ulrich, A., and Hallmann, J. 2005. Endophytic and ectophytic potato-associated bacterial communities differ in structure and antagonistic function against plant pathogenic fungi. FEMS (Fed. Eur. Microbiol. Soc.) Microbiol. Ecol. 51:215-229.

Blankenberg, D., Von Kuster, G., Coraor, N., Ananda, G., Lazarus, R., Mangan, M., Nekrutenko, A., and Taylor, J. 2010. Galaxy: A Web-based genome analysis tool for experimentalists. Curr. Protocols Mol. Biol. 19.10:1-21.

Bodenhausen, N., Horton, M. W., and Bergelson, J. 2013. Bacterial communities associated with the leaves and the roots of Arabidopsis thaliana. PLoS One 8:e56329. Published online.

Bokulich, N. A., Subramanian, S., Faith, J. J., Gevers, D., Gordon, J. I., Knight, R., Mills, D. A., and Caporaso, J. G. 2013. Quality-filtering vastly improves diversity estimates from Illumina amplicon sequencing. Nat. Methods 10:57-59.

Bouttier, C., and Morgan, D. G. 1992. Development of soilseed rape buds, flowers and pods in vitro. J. Exp. Bot. 43:1089-1096.

Brandt, B. W., Bonder, M. J., Huse, S. M., and Zaura, E. 2012. TaxMan: A server to trim rRNA reference databases and inspect taxonomic coverage. Nucleic Acids Res. 40:82-87.

Bulgarelli, D., Rott, M., Schlaeppi, K., Ver Loren van Themaat, E., Ahmadinejad, N., Assenza, F., Rauf, P., Huettel, B., Reinhardt, R., Schmelzer, E., Peplies, J., Gloeckner, F. O., Amann, R., Eickhorst, T., and Schulze-Lefert, P. 2012. Revealing structure and assembly cues for Arabidopsis root-inhabiting bacterial microbiota. Nature 488:91-95.

Bull, C. T., Boer, S. H. De, Denny, T. P., Firrao, G., Saux, M. F., Saddler, G. S., Scortichini, M., Stead, D. E., and Takikawa, Y. 2010. Comprehensive list of names of plant pathogenic bacteria, 1980-2007. J. Plant Pathol. 92:551-592.

Caporaso, J. G., Bittinger, K., Bushman, F. D., DeSantis, T. Z., Andersen, G. L., and Knight, R. 2010. PyNAST: A flexible tool for aligning sequences to a template alignment. Bioinformatics 26:266-267.

Chakravorty, S., Helb, D., Burday, M., and Connell, N. 2008. NIH Public Access 69:330-339.

Chelius, M. K., and Triplett, E. W. 2001. The Diversity of Archaea and Bacteria in Association with the Roots of Zea mays L. Microb. Ecol. 41:252-263.

Compant, S., Duffy, B., Nowak, J., and Cle, C. 2005. Use of plant growthpromoting bacteria for biocontrol of plant diseases: principles, mechanisms of action, and future prospects. Appl. Environ. Microbiol. 71:4951-4959.

De Oliveira Costa, L., de Queiroz, M., Borges, A., de Moraes, C., and de Araujo, E. 2012. Isolation and characterization of endophytic bacteria isolated from the leaves of the common bean (Phaseolus vulgaris). Braz. J. Microbiol. 43:1562-1575.

DeSantis, T. Z., Hugenholtz, P., Larsen, N., Rojas, M., Brodie, E. L., Keller, K., Huber, T., Dalevi, D., Hu, P., and Andersen, G. L. 2006. Greengenes, a chimera-checked 16S rRNA gene database and workbench compatible with ARB. Appl. Environ. Microbiol. 72:5069-5072.

Edgar, R. C. 2010. Search and clustering orders of magnitude faster than BLAST. Bioinformatics 26:2460-2461.

Finkel, O. M., Burch, A. Y., Lindow, S. E., Post, A. F., and Belkin, S. 2011. Geographical location determines the population structure in phyllosphere microbial communities of a salt-excreting desert tree. Appl. Environ. Microbiol. 77:7647-7655.

Giardine, B., Riemer, C., Hardison, R. C., Burhans, R., Shah, P., Zhang, Y., Blankenberg, D., Albert, I., Miller, W., Kent, W. J., and Nekrutenko, A. 2005. Galaxy: A platform for interactive large-scale genome analysis. Cold Spring Harb. Lab. Press 15:1451-1455.

Gionis, A., and Mannila, H. 2007. Assessing data mining results via swap randomization. ACM Trans. Knowl. Discov. Data 1:1401-1432.

Goecks, J., Nekrutenko, A., and Taylor, J. 2010. Galaxy: A comprehensive approach for supporting accessible, reproducible, and transparent computational research in the life sciences. Genome Biol. 11:R86.

Gomez-Gomez, L., Felix, G., and Boller, T. 1999. A single locus determines sensitivity to bacterial flagellin in Arabidopsis thaliana. Plant J. $18: 277-284$..

Gunasinghe, U. B., Irwin, M. E., and Kampmeier, G. E. 1988. Soybean leaf pubescence affects aphid vector transmission and field spread of soybean mosaic virus. Ann. Appl. Biol. 112:259-272. 
Hardoim, P. R., Hardoim, C. C. P., van Overbeek, L. S., and van Elsas, J. D. 2011. Dynamics of seed-borne rice endophytes on early plant growth stages. PLoS One 7:e30438. Published online.

Innerebner, G., Knief, C., and Vorholt, J. A. 2011. Protection of Arabidopsis thaliana against leaf-pathogenic Pseudomonas syringae by Sphingomonas strains in a controlled model system. Appl. Environ. Microbiol. 77:3202-3210.

Jackson, C. R., and Denney, W. C. 2010. Annual and seasonal variation in the phyllosphere bacterial community associated with leaves of the southern Magnolia (Magnolia grandiflora). Microb. Ecol. 61:113122

Johnson, H. B. 1975. Plant pubescence: An ecological perspective. Bot. Rev. 41:233-258. 2012

Kim, M., Singh, D., Lai-Hoe, A., Go, R., Abdul Rahim, R., Ainuddin, A N., Chun, J., and Adams, J. M. 2012. Distinctive phyllosphere bacterial communities in tropical trees. Microb. Ecol. 63:674-681.

Knief, C., Ramette, A., Frances, L., Carlos, A.-B., and Vorholt, J. A. 2010. Site and plant species are important determinants of the Methylobacterium community composition in the plant phyllosphere. ISME J. 4:719728.

Kuczynski, J., Liu, Z., Lozupone, C., McDonald, D., Fierer, N., and Knight, R. 2010. Microbial community resemblance methods differ in their ability to detect biologically relevant patterns. Nat. Methods 7:813-819.

Kuklinsky-Sobral, J., Araújo, W. L., Mendes, R., Geraldi, I. O., PizziraniKleiner, A. A., Azevedo, J. L., Júlia, K.-S., and A, Pizzirani-Kleiner, A 2004. Isolation and characterization of soybean-associated bacteria and their potential for plant growth promotion. Environ. Microbiol. 6:12441251.

Langille, M. G. I., Zaneveld, J., Caporaso, J. G., McDonald, D., Knights, D., Reyes, J. A., Clemente, J. C., Burkepile, D. E., Vega Thurber, R. L., Knight, R., Beiko, R. G., and Huttenhower, C. 2013. Predictive functional profiling of microbial communities using 16S rRNA marker gene sequences. Nat. Biotechnol. 31:814-821.

Lindow, S. E., and Brandl, M. T. 2003. Microbiology of the phyllosphere. Appl. Environ. Microbiol. 69:1875-1883.

Lozupone, C., and Knight, R. 2005. UniFrac: A new phylogenetic method for comparing microbial communities. Appl. Environ. Microbiol. 71:8228-8235.

Lozupone, C., Lladser, M. E., Knights, D., Stombaugh, J., and Knight, R. 2011. UniFrac: An effective distance metric for microbial community comparison. ISME J. 5:169-172.

Madhaiyan, M., Suresh Reddy, B. V, Anandham, R., Senthilkumar, M., Poonguzhali, S., Sundaram, S. P., and Sa, T. 2006. Plant growth-promoting Methylobacterium induces defense responses in groundnut (Arachis hypogaea L.) compared with rot pathogens. Curr. Microbiol. 53:270-276.

Mahuku, G. S., Goodwin, P. H., Hall, R., and Hsiang, T. 1997. Variability in the highly virulent type of Leptosphaeria maculans within and between oilseed rape fields. Can. J. Bot. 75:1485-1492.

Maignien, L. L., DeForce, E. A., Chafee, M. E., Eren, A. M., and Simmons, S. L. 2014. Ecological succession and stochastic variation in the assembly of Arabidopsis thaliana phyllosphere communities. MBio 5:e00682-13. Published online.

Maughan, H., Wang, P. W., Diaz Caballero, J., Fung, P., Gong, Y., Donaldson, S. L., Yuan, L., Keshavjee, S., Zhang, Y., Yau, Y. C. W., Waters, V. J., Tullis, D. E., Hwang, D. M., and Guttman, D. S. 2012. Analysis of the cystic fibrosis lung microbiota via serial Illumina sequencing of bacterial $16 \mathrm{~S}$ rRNA hypervariable regions. PLoS One 7:e45791. Published online.

McDonald, D., Price, M. N., Goodrich, J., Nawrocki, E. P., DeSantis, T. Z., Probst, A., Andersen, G. L., Knight, R., and Hugenholtz, P. 2012. An improved Greengenes taxonomy with explicit ranks for ecological and evolutionary analyses of bacteria and archaea. ISME J. 6:610-618.

Peiffer, J. A., Spor, A., Koren, O., Jin, Z., Green, S., and Dangl, J. L. 2013. Diversity and heritability of the maize rhizosphere microbiome under field conditions. Proc. Natl. Acad. Sci. U.S.A. 110:6548-6553.

Perazzolli, M., Antonielli, L., Storari, M., Storari, M., Puopolo, G., Pancher, M., Giovannini, O., Pindo, M., and Pertot, I. 2014. Resilience of the natural phyllosphere microbiota of the grapevine to chemical and biological pesticides. Appl. Environ. Microbiol. 80:3585-9356.

Price, M. N., Dehal, P. S., and Arkin, A. P. 2009. FastTree: Computing large minimum evolution trees with profiles instead of a distance matrix. Mol. Biol. Evol. 26:1641-1650.

Rajendran, G., Patel, M. H., and Joshi, S. J. 2012. Isolation and Characterization of nodule-associated Exiguobacterium sp. from the root nodules of fenugreek (Trigonella foenum-graecum) and their possible role in plant growth promotion. Int. J. Microbiol. 2012:e693982.

Rastogi, G., Sbodio, A., Tech, J. J., Suslow, T. V, Coaker, G. L., and Leveau, J. H. J. 2012. Leaf microbiota in an agroecosystem: Spatiotemporal variation in bacterial community composition on field-grown lettuce. ISME J. 6:1812-1822.

Rastogi, G., Coaker, G. L., and Leveau, J. H. 2013. New insights into the structure and function of phyllosphere microbiota through highthroughput molecular approaches. FEMS (Fed. Eur. Microbiol. Soc.) Microbiol. Lett. 348:1-10.

Redford, A. J., and Fierer, N. 2009. Bacterial succession on the leaf surface: A novel system for studying successional dynamics. Microb. Ecol. 58:189-198.

Redford, A. J., Bowers, R. M., Knight, R., Linhart, Y., and Fierer, N. 2010. The ecology of the phyllosphere: Geographic and phylogenetic variability in the distribution of bacteria on tree leaves. Environ. Microbiol. 12:2885-2893.

Schloss, P. D., and Handelsman, J. 2006. Toward a census of bacteria in soil. PLoS Comput. Biol. 2:e92. Published online.

Schloss, P. D., Westcott, S. L., Ryabin, T., Hall, J. R., Hartmann, M., Hollister, E. B., Lesniewski, R. A., Oakley, B. B., Parks, D. H., Robinson, C. J., Sahl, J. W., Stres, B., Thallinger, G. G., Van Horn, D. J., and Weber, C. F. 2009. Introducing Mothur: Open-source, platform-independent, community-supported software for describing and comparing microbial communities. Appl. Environ. Microbiol. 75:7537-7541.

Shade, A., McManus, P. S., and Handelsman, J. 2013. Unexpected diversity during community succession in the apple flower microbiome. MBio 4:602-612

Tatusov, R. L., Galperin, M. Y., Natale, D. A, and Koonin, E. V. 2000. The COG database: A tool for genome-scale analysis of protein functions and evolution. Nucleic Acids Res. 28:33-36.

Van Dongen, S. 2000. Graph clustering by flow simulation. Ph.D. thesis, University of Utrecht, The Netherlands.

Vorholt, J. A. 2012. Microbial life in the phyllosphere. Nat. Rev. Microbiol. 10:828-840.

Wang, Q., Garrity, G. M., Tiedje, J. M., and Cole, J. R. 2007. Naive Bayesian classifier for rapid assignment of rRNA sequences into the new bacterial taxonomy. Appl. Environ. Microbiol. 73:5261-5267.

Wang, Y., and Qian, P.-Y. 2009. Conservative fragments in bacterial 16S rRNA genes and primer design for $16 \mathrm{~S}$ ribosomal DNA amplicons in metagenomic studies. PLoS One 4:e7401. Published online.

Zhang, S. Z., Xue, A. G., Zhang, J. X., Cober, E., Anderson, T. R., Poysa, V., and Rajcan, I. 2010. Reactions of Canadian short-season soybean cultivars to three races of Phytophthora sojae. Can. J. Plant Sci. 90:207210 .

\section{AUTHOR-RECOMMENDED INTERNET RESOURCE}

HIV sequence database: www.hiv.lanl.gov 